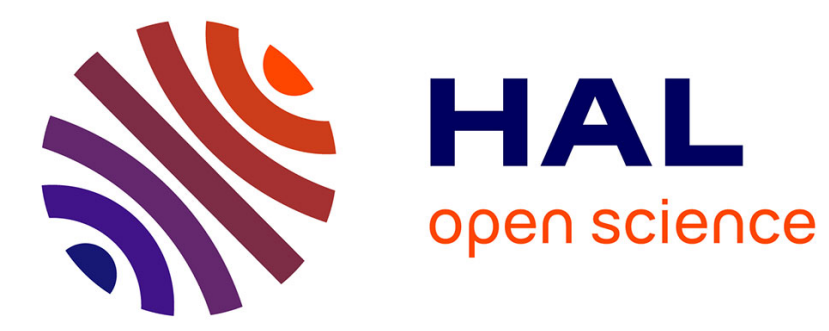

\title{
Shear with comminution of a granular material: Microscopic deformations outside the shear band
}

Guillaume Chambon, Jean Schmittbuhl, Alain Corfdir, Jean-Pierre Vilotte, Stéphane Roux

\section{To cite this version:}

Guillaume Chambon, Jean Schmittbuhl, Alain Corfdir, Jean-Pierre Vilotte, Stéphane Roux. Shear with comminution of a granular material: Microscopic deformations outside the shear band. Physical Review E , 2003, 68 (1), 10.1103/PhysRevE.68.011304 . hal-01982360

\section{HAL Id: hal-01982360 https://hal.science/hal-01982360}

Submitted on 28 Jan 2021

HAL is a multi-disciplinary open access archive for the deposit and dissemination of scientific research documents, whether they are published or not. The documents may come from teaching and research institutions in France or abroad, or from public or private research centers.
L'archive ouverte pluridisciplinaire HAL, est destinée au dépôt et à la diffusion de documents scientifiques de niveau recherche, publiés ou non, émanant des établissements d'enseignement et de recherche français ou étrangers, des laboratoires publics ou privés. 


\title{
Shear with comminution of a granular material: Microscopic deformations outside the shear band
}

\author{
G. Chambon and J. Schmittbuhl \\ Laboratoire de Géologie, UMR CNRS 8538, École Normale Supérieure, 24, rue Lhomond, 75231 Paris Cédex 05, France
}

A. Corfdir

CERMES, ENPC/LCPC, Institut Navier, 6 et 8 avenue Blaise Pascal, 77455 Champs sur Marne Cédex 2, France

J. P. Vilotte

Département de Sismologie, UMR CNRS 7580, Institut de Physique du Globe de Paris, 4 place Jussieu, 75252 Paris Cédex 05, France

S. Roux

Laboratoire de Surface du Verre et Interfaces, CNRS/St-Gobain, quai L. Lefranc, 93303 Aubervilliers Cédex, France

(Received 27 March 2003; published 23 July 2003)

\begin{abstract}
A correlation imaging velocimetry technique is applied to recover displacement fields in a granular material subjected to extended shear. A thick $(10 \mathrm{~cm})$ annular sand sample (grain size: $1 \mathrm{~mm}$ ) is confined at constant pressure $(\sigma=0.5 \mathrm{MPa})$ against a rough moving wall displacing at very low speed $\left(\dot{\delta}=83 \mu \mathrm{m} \mathrm{s}^{-1}\right)$. Localization of the strain rapidly forms a shear band (seven particles wide) in which comminution develops. We focused on the strain field outside this shear band and observed a rich dynamics of large and intermittent mechanical clusters (up to 50 particles wide). Quantitative description of the radial velocity profile outside the shear band reveals an exponential decrease. However, a significant slip evolution of the associated characteristic length is observed, indicative of a slow decoupling between the shear band and the rest of the sample. This slow evolution is shown to be well described by power laws with the imposed slip, and has important implications for friction laws and earthquake physics.
\end{abstract}

DOI: 10.1103/PhysRevE.68.011304 PACS number(s): 83.80.Fg, 62.20.Fe, 83.10.Pp, 91.30.Bi

\section{INTRODUCTION}

Shear processes in granular materials are described experimentally from two very different approaches. Most of the studies concern friction laws, i.e., evolutions of the shear strength with slip history at a macroscopic scale [1-3]. Local processes are averaged over the whole interface and a constitutive law is proposed. A second approach focuses on observations of local processes such as displacement fields and velocity profiles [4-6], or stress field using photoelasticity [7]. Experimental results are often compared to discrete element simulations, which usually provide higher spatial and temporal resolution [8-10]. In particular, numerous studies have addressed the properties of velocity profiles during shear in Couette configuration. However, little work has been performed on the prolongation of these profiles far from the shear interface. Similarly, the influence of comminution on the granular flow remains mostly unknown, though very relevant for the shearing of angular particles.

In this paper, we report on recent results obtained from the analysis of strain fields during shear of an assembly of angular sand grains. Using an annular pseudo-Couette apparatus, we explore the evolution of the strain field over very large slips (several meters). Because of angular shapes of the particles, crushing exists inside a shear band and strongly influence the behavior of the material. In particular, we study extensively the large scale region surrounding the shear band. This region appears marked by very slow and rich dynamics. The azimuthal velocity field established after localization displays exponential radial profiles, with a charac- teristic penetration length that progressively decreases as imposed slip advances. This relaxation with slip denotes a slow decoupling between the shear band and the rest of the sample, which we interpret as a consequence of comminution inside the band. We relate this slow evolution with slip of the local strain to the recently observed large (seismiclike) slip weakening of the macroscopic shear strength [11].

\section{EXPERIMENTAL SETUP}

As sketched in Fig. 1, we use a pseudo-Couette shear apparatus in which an annular sample of granular material (square cross section) is confined between an inner steel cylinder and an outer neoprene jacket [12]. The cylinder is rotated at a prescribed angular velocity of 0.83 $\times 10^{-3} \mathrm{rads}^{-1}$, which corresponds to a linear velocity $\dot{\delta}$ $=83 \mu \mathrm{m} \mathrm{s}^{-1}$ at the surface of the inner cylinder. A constant confining pressure $\sigma=500 \mathrm{kPa}$ is applied through the jacket. Vertically, the sample is embedded between a glass plate and a rigid upper lid made of dural. Triangular grooves machined on the cylinder surface perpendicular to the sliding direction insure good transmission of the strain to the sample.

Results presented in this paper have been obtained with an angular quartz sand sieved between 0.80 and $1.25 \mathrm{~mm}$ (distribution mode: $1 \mathrm{~mm}$ ). Samples are prepared by pouring the material into the apparatus in successive layers. Each layer is gently compacted by hand-applied vibrations. This protocol results in relatively dense samples with an intial porosity ranging between $40 \%$ and $48 \%$. The samples typi- 


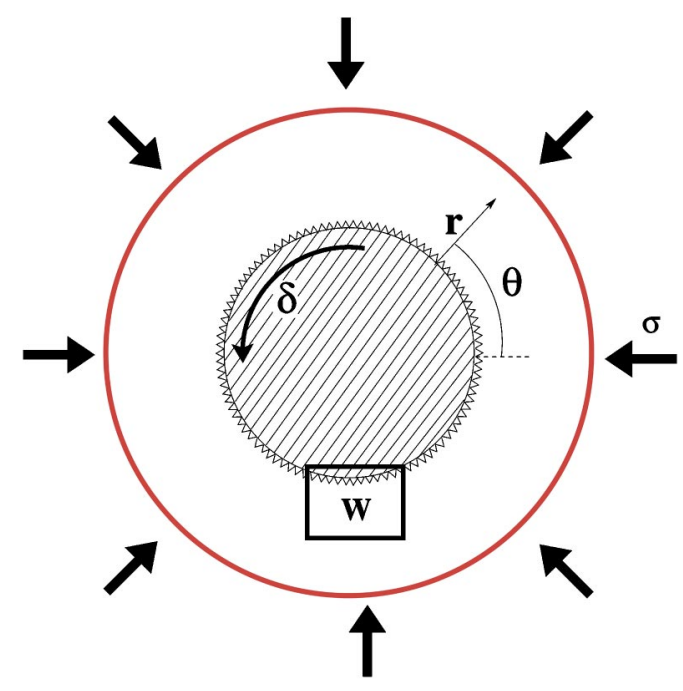

FIG. 1. Sketch of the annular simple shear apparatus (ACSA). The granular sample presents a cylindrical symmetry with an annular horizontal section. The inner rotating cylinder has a radius of $R=10 \mathrm{~cm}$. The outside jacket is a cylinder of radius $20 \mathrm{~cm}$ and is submitted to a normal pressure $\sigma=0.5 \mathrm{MPa}$. The vertical dimension of the sample is $10 \mathrm{~cm}$. Position inside the sample is defined by using cylindrical coordinates $(r, \theta, z)$, with the origin of radius $r$ taken at the cylinder surface. The artificial roughness of the inner cylinder is magnified (1-mm-deep grooves). The observation window $\mathbf{W}$ is figured.

cally exhibit dilatancy during the first increments of shear strain.

Direct observation of the granular sample while shearing is enabled by a window pierced in the apparatus bottom plate (Fig. 1). Series of digital photographs are taken through this window using a KODAK DCS 420 camera (resolution: $1536 \times 1024$ px, 8 bit gray levels) with a NIKON $35 \mathrm{~mm}$ macrolens. Examples of digital images are shown in Fig. 2.

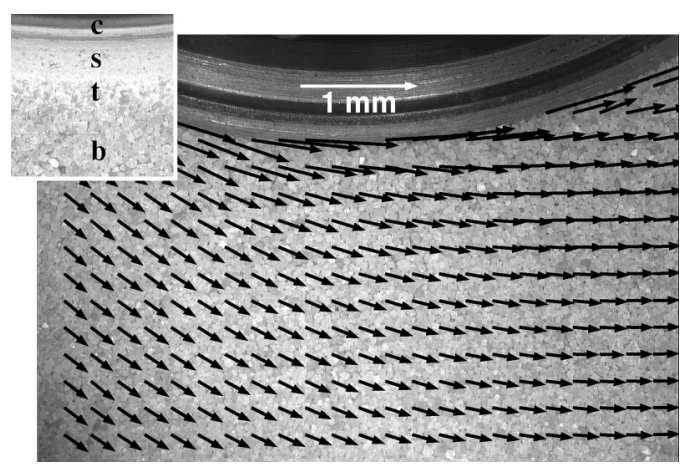

FIG. 2. Background is a raw digital picture taken through the observation window. It corresponds to an imposed slip $\delta$ $=2.5 \mathrm{~mm}$. Superimposed vectors represent the incremental displacement field du in the sample. It is computed by applying CIV between this photo and the following one (taken in a 10-s delay). Subregions for correlation computation size $64 \times 64 \mathrm{px}$. The white arrow gives the scale of the plot. A detail of the shear band that develops after localization is included in the upper left corner of the image: $c$, rotating cylinder; $s$, shear band; $t$, transition layer; $b$, bulk, where original particles can be observed.

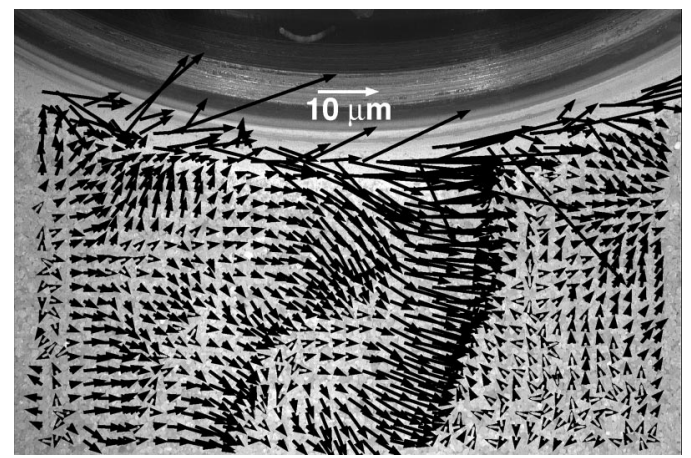

FIG. 3. Same plot as in Fig. 2, but for a pair of photos taken at larger $\delta$, namely, $320 \mathrm{~mm}$. Note the considerable scale magnification. Typical incremental displacements outside the shear band are here about $10 \mu \mathrm{m}$, that is, a hundredth of the imposed slip increment $\Delta \delta$. Inside the shear band, computed displacement are erratic and not reliable.

They comprise a small portion of the inner cylinder and about $110 \times 60$ sand grains. A single grain typically covers a surface area of 150 pixels. In the following, our data will essentially come from one series of 400 pictures displaying sufficient contrast and sharpness. Time interval between two successive photographs is $10 \mathrm{~s}$, which corresponds to a slip increment of the rotating cylinder of $\Delta \delta=0.83 \mathrm{~mm}$. The series begins at the onset of shear (fresh sample) and covers about $35 \mathrm{~cm}$ of slip. Other series recorded in similar conditions have also been studied to check the reproducibility of our results.

Digital photographs are analyzed by means of correlation imaging velocimetry (CIV) in order to recover grain motions inside the sample (see Appendix). This technique yields a two-dimensional (2D) local displacement field $\mathbf{u}(r, \theta)$, coarse grained over small subregions. A typical output of CIV is shown in Fig. 2. Note that we do not recover the displacement of each individual grain, but an averaged value over subregions, which typically comprises $4.5 \times 4.5$ particles. As discussed in the Appendix, the accuracy achieved in displacement determination is about $2 \mu \mathrm{m}$. Obviously, the technique does not account for possible off-plane (vertical) components of grain motions. We checked, however, that these components are sufficiently small in our experiments not to affect the determination of horizontal displacements. (No grain ever "disappeared" from a series of photos.)

\section{RESULTS}

\section{A. Strain heterogeneities inside the bulk}

We consider here the incremental displacement fields du (proportional to velocity $\mathbf{v}$ ) calculated between each pair of successive photos. Comparison of Figs. 2 and 3 clearly shows localization of deformation: The magnitude of incremental displacements inside the sample has dramatically dropped during the slip interval between these two figures. Actually, localization occurs during the very first millimeters of slip [13]. For values of $\delta$ typically larger than $10 \mathrm{~mm}$, most of the prescribed slip is already accommodated in a seven-grain wide interfacial layer around the inner cylinder, 
i.e., in a shear band (see inset of Fig. 2). Due to the confining pressure and the angularity of the used sand, grains undergo intense crushing inside this band [layer (s) in Fig. 2]. As a consequence, CIV is inapplicable for this region of the sample (Fig. 3).

On the contrary, the CIV technique is very well suited outside the interfacial layer, i.e., in the bulk of the sample. Though very small compared to the cylinder slip increment $\Delta \delta$, incremental displacements inside the bulk are well resolved (Fig. 3). Such small displacements (they may reach $50 \mu \mathrm{m}$ at most, i.e., a few hundredths of the mean grain size) presumably correspond to local vibrations of the grains. The displacement field displays strong spatial heterogeneities. Large patches of coherent displacement are commonly observed, whose extension might reach the window size (50 grains). These zones seem characterized by rigid motion of the particles and are probably the hallmark of mechanical clusters. They are completely invisible from a direct observation of the particle assembly (no associated microstructure). Also, these patches appear strongly intermittent on the time basis we used $(0.1 \mathrm{~Hz})$ : very little persistence is observed when comparing consecutive displacement fields.

Raw displacement fields may involve a small but unknown artifactual component due to possible vibrations of the camera because of motor noise or ongoing comminution, slow creeping move of the camera tripod, etc. Nevertheless, this experimental shift is expected to be homogeneous in space. It is thus eliminated when computing the incremental strain tensor classically defined as $\underline{d \varepsilon}=(1 / 2)\left(\underline{d G}+\underline{d G^{T}}\right)$, where $\underline{d G}=\boldsymbol{\nabla} \otimes$ du. In Fig. 4, we show the incremental volumetric strain $d \varepsilon_{V}$ and incremental shear strain $d \gamma$ derived from the displacement field presented in Fig. 3. These fields are, respectively, defined as $d \varepsilon_{V}=d \varepsilon_{1}+d \varepsilon_{2}$ and $d \gamma$ $=\left|d \varepsilon_{1}-d \varepsilon_{2}\right|$, where $d \varepsilon_{1}$ and $d \varepsilon_{2}$ are the two eigenvalues of the tensor $\underline{d \varepsilon}$.

The large scale patches observed in the displacement field appear as deformation bands characterized by compaction or dilatancy and large shear (Fig. 4). These bands are generally inclined with respect to the slip direction. Note that because of the finite size of the CIV subregions, a significant smoothing of the strain fields is introduced, associated with a spatial spreading of the structures. Specifically, dilatancy and compaction bands seem typically associated with the boundaries of the mechanical clusters. This confirms that the sample behaves as an assembly of quasirigid regions, which may be analogous to eddylike structures observed in Ref. [14]. Furthermore, despite their very short life, it is sometimes possible to observe that these bands are emitted by the interfacial layer, and then propagate into the bulk such as avalanche events.

\section{B. Exponential radial velocity profiles}

We now study the radial profiles of incremental displacements observed inside the bulk after localization (Fig. 5). Though less precisely resolved than strain, incremental displacements (and velocity) are at first easier to interpret. We will focus on azimuthal displacements, which are always greater in magnitude and less affected by artifacts than radial

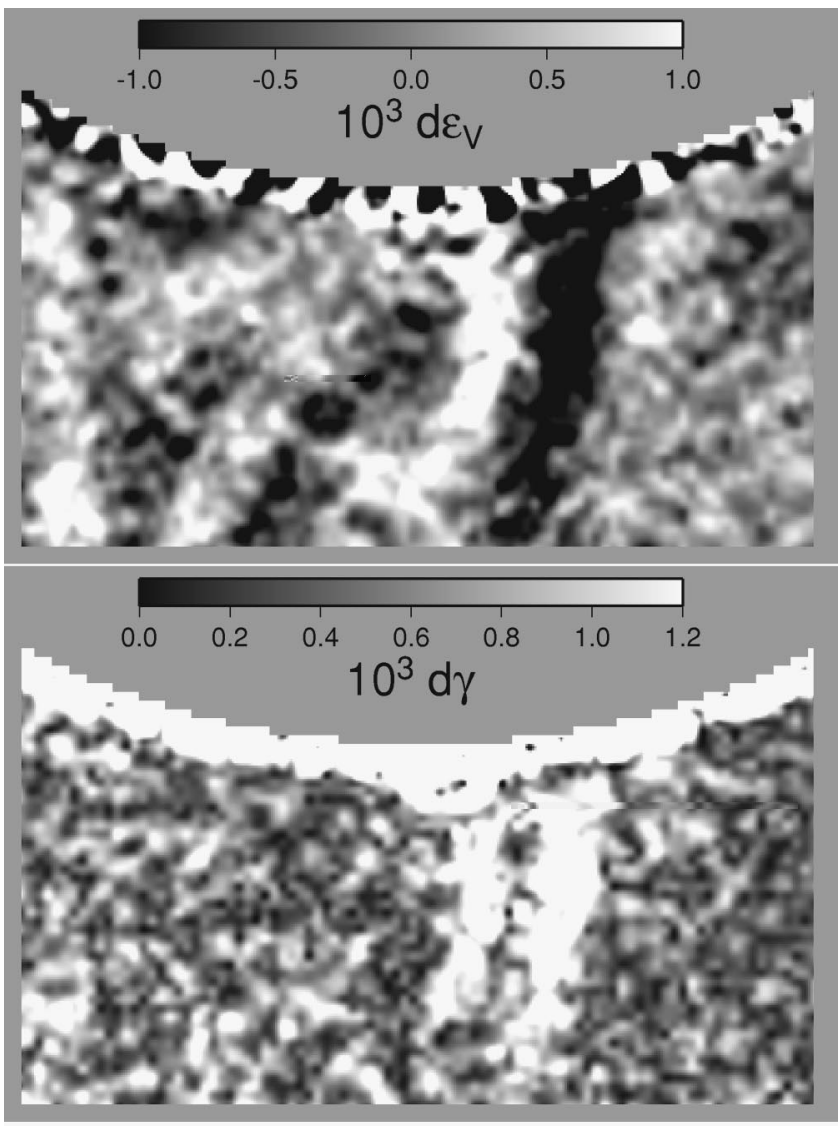

FIG. 4. Incremental volumetric (upper panel) and shear (lower panel) strains $d \varepsilon_{V}$ and $d \gamma$ estimated from the incremental displacement field shown in Fig. 3. Positive $d \varepsilon_{V}$ indicates dilation, negative $d \varepsilon_{V}$ indicates compaction.

displacements. To reduce spatial and time fluctuations, the presented profiles are averaged azimuthally as well as over small $\delta$ windows. It appears that, above its detection threshold, the azimuthal component $d u_{\theta}$ is reasonably well characterized by an exponential decay with $r$ [Fig. 5(a)]:

$$
d u_{\theta}=C_{0}(\delta) e^{-r / \lambda(\delta)} \Delta \delta .
$$

In general, the prefactor $C_{0}$ should be regarded as $\delta$ dependent. Similarly, and despite significant fluctuations, the penetration length $\lambda$ of the profiles clearly displays a decreasing trend with slip $\delta$ [Fig. 5(b)]. Extremal values of $\lambda$ are about $9 \mathrm{~mm}$ for small $\delta$, and $3 \mathrm{~mm}$ for large $\delta$.

Various studies have documented, in shear cells, the azimuthal velocity profile established within the first ten grain layers against the moving wall (i.e., within the shear band) $[5,6]$. It reproducibly consists in the combination of a decreasing exponential and a Gaussian. Here, we show that this profile is prolongated by an exponential tail outside the interfacial layer. Interestingly, such an exponential tail, associated with highly heterogeneous and intermittent clusters, is reminiscent of the small creep motion observed in the "static" part of avalanching piles [15,16]. Particularity of our situation, however, is the slow evolution of this creep, as denoted by the progressive decrease in $\lambda(\delta)$. 


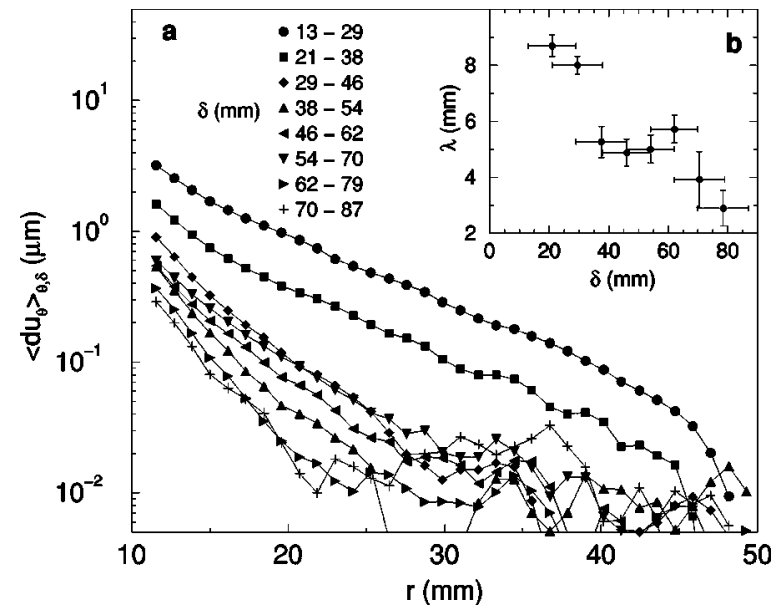

FIG. 5. (a) Evolution of azimuthal component $d u_{\theta}$ of incremental displacements, as a function of radius $r$ and for eight values of slip $\delta$ (semilogarithmic plot). The profiles are averaged over all the resolved angles $\theta$, and over small $\delta$ windows detailed in the legend. The asymptotic limit at large $\delta$ of all the profiles has been artificially set to 0 . On the raw data, this saturation value was finite, but always below the CIV detection threshold. (b) Evolution of the penetration length $\lambda$ (see text) as a function of slip $\delta$. Values of $\lambda(\delta)$ are calculated from linear regressions of the profiles displayed in (a). Vertical error bars represent the range of possible results depending on the chosen fitting region.

\section{A logarithmic slip relaxation}

In Fig. 6, we show the postlocalization slip evolution of averaged incremental shear and volumetric strains. The represented curves correspond to a zone of the bulk relatively close to the interfacial layer ( $\operatorname{small} r$ ). Both quantities show a transient regime over typically the first $50 \mathrm{~mm}$ of slip after localization. Shear strain decreases in magnitude by a factor of 4, while volumetric strain exhibits a transition from a strongly compactant toward a quasineutral regime. This slow relaxation of incremental strains can be seen as a consequence of the progressive steepening of velocity profiles inside the bulk (Fig. 5). Significant fluctuations during the sta-

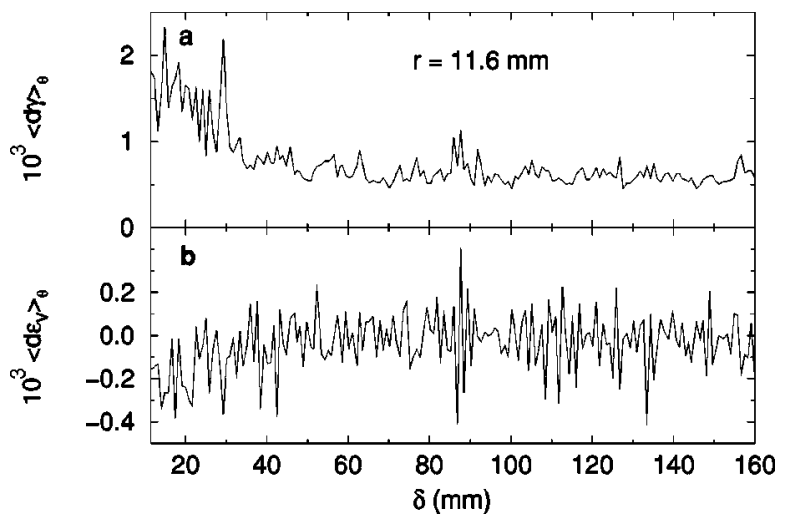

FIG. 6. Evolution of the azimuthally averaged incremental shear and the volumetric strains $\langle d \gamma\rangle_{\theta}$ and $\left\langle d \varepsilon_{V}\right\rangle_{\theta}$ as a function of slip $\delta$, for $r=11.6 \mathrm{~mm}$. The plot begins at $\delta=11.5 \mathrm{~mm}$, i.e., after localization.

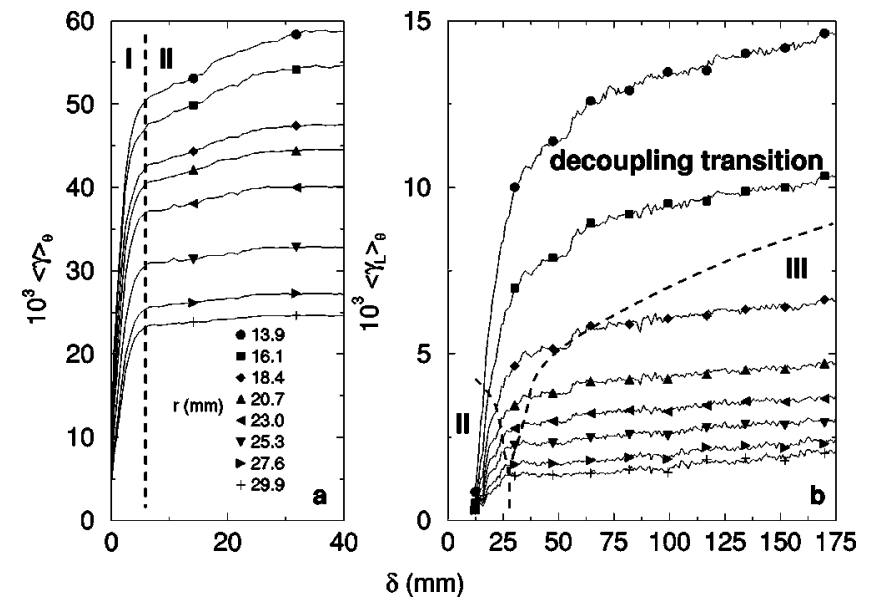

FIG. 7. Evolution of the azimuthally averaged cumulative shear strain as a function of slip $\delta$, for eight values of $r$ inside the bulk (see legend). For this plot only, the size of the subregions used in CIV computations is $128 \times 128 \mathrm{px}$. (a) Cumulative shear strain from initial state $\langle\gamma\rangle_{\theta}$. (b) Cumulative shear strain since localization $\left\langle\gamma_{L}\right\rangle_{\theta}$. The dashed lines have been traced qualitatively to indicate the transitions between the four deformation regimes (see text).

tionnary regime at large $\delta$ illustrate the strong intermittency of incremental displacements and strains in the bulk.

It can be seen in Fig. 5(a) that, as soon as $\delta>30 \mathrm{~mm}$, incremental quantities emerge from the noise level only for $r<20-30 \mathrm{~mm}$, typically. To resolve for larger radii, cumulative quantities have to be computed. We determined two different cumulative displacement fields. The first, denoted $\mathbf{u}(r, \theta ; \delta)$, is calculated by applying CIV between the first picture of the series (reference is the fresh sample) and all the following pictures. The second, denoted $\mathbf{u}_{L}$, is calculated from a reference picture corresponding to $\delta=12 \mathrm{~mm}$ and thus represents cumulative displacements since localization. The field $\mathbf{u}_{L}$ can be considered as a refinement of the field $\mathbf{u}$ for postlocalization. Indeed, CIV accuracy significantly decreases when the magnitude of the searched displacement vectors reaches about one-fourth of the used subregion size [17] — a situation met even before localization in our shear experiments.

Averaged cumulative shear strains $\langle\gamma\rangle_{\theta}$ and $\left\langle\gamma_{L}\right\rangle_{\theta}$ derived from the fields $\mathbf{u}$ and $\mathbf{u}_{L}$ are presented as a function of slip in Fig. 7. Here, we focus on postlocalization [Fig. 7(b)]. For small values of $r$, the slope of the curve $\left\langle\gamma_{L}\right\rangle_{\theta}(\delta)$ slowly decreases with $\delta$, consistent with the slow decrease of the increment $\langle d \gamma\rangle_{\theta}(\delta)$ in Fig. 6. When $r$ increases, besides an overall reduction in magnitude, the shape of the $\delta$ profiles also displays a notable evolution. From smooth at small $r$, the transition between high and low strain increments progressively sharpens with $r$. For the highest displayed $r$ values, this transition seems to occur quasi-instantaneously, between two linear regimes, at $\delta \approx 26 \mathrm{~mm}$ [Fig. 7(b)].

A more quantitative assessment of this sharpening process is proposed in Fig. 8. As shown, the slip evolution of $\left\langle\gamma_{L}\right\rangle_{\theta}$ for small values of $r$ is very consistent with a logarithmic increase:

$$
\gamma_{L} \propto \ln \left(\delta_{L} / \delta^{*}\right)
$$




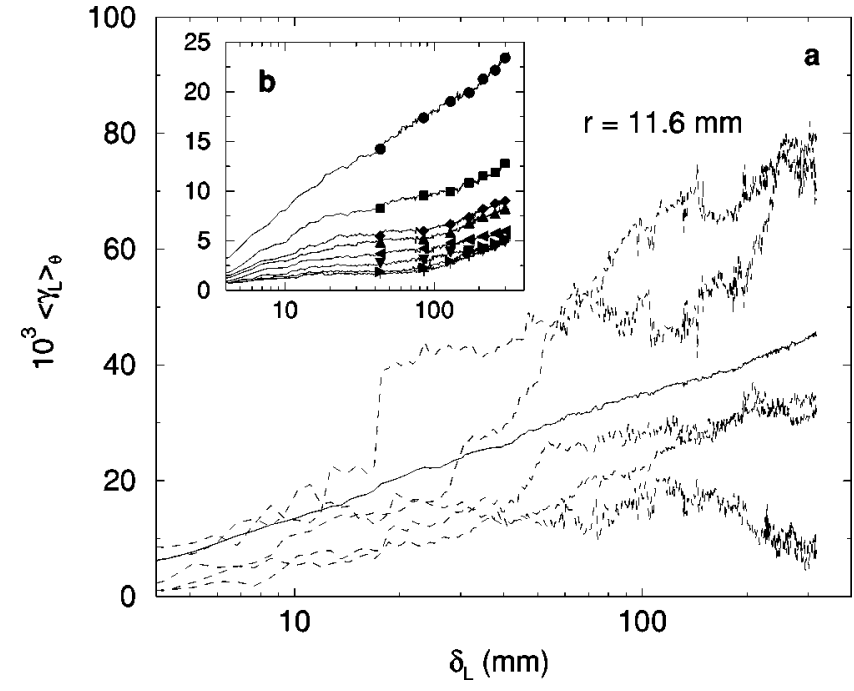

FIG. 8. Slip evolution of the azimuthally averaged cumulative shear strain $\left\langle\gamma_{L}\right\rangle_{\theta}$ in semilogarithmic plot. Origin for slip definition is here taken at localization: $\delta_{L}=\delta-12 \mathrm{~mm}$. (a) Comparison between the azimuthal average for $r=11.6 \mathrm{~mm}$ (solid curve) and five local (nonaveraged) data sets picked on the corresponding azimuthal profile (dashed curves). (b) Comparison between eight different values of $r$ inside the bulk. Symbols are the same as in Fig. 7.

where slip $\delta_{L}$ is counted from localization, and $\delta^{*}$ is a normalizing factor. For larger values of $r$, on the contrary, data show pronounced negative curvature in semi-log scales [Fig. 8(b)], indicative of a faster decrease of the increments with $\delta$. Hence, though postlocalization relaxation of strain increments can be observed in the whole bulk, the duration of this process clearly changes when $r$ increases. Slow, logarithmic relaxation only exists close to the interfacial layer, typically up to $r=20-30 \mathrm{~mm}$. Furthermore, even for small values of $r$, the slip evolution of the nonaveraged shear strain $\gamma_{L}(r, \theta)$ generally significantly departs from logarithmic [Fig. 8(a)]. This local quantity displays large fluctuations that tend to grow with slip. Thus, slow relaxation, when it exists, emerges only from an averaging in space. Local processes are much sharper and strongly heterogeneously distributed.

If the cumulative strain evolves logarithmically for small $r$, then the incremental strain relaxes following a hyperbolic law:

$$
d \gamma \propto \delta^{* /} \delta .
$$

From Fig. 6, the shear strain increments are clearly expected to reach a nonzero limit for large values of $\delta$. The above relaxation law (3) should thus be restricted to small slips only. Nevertheless, this empirical law is important since it clearly establishes that the slow relaxation we observe at small $r$ [and thus the decrease of $\lambda(\delta)$ ] does not involve any characteristic slip scale.

\section{Proposed modeling}

We now attempt to combine both results (1) and (2) into a consistent expression for postlocalization slow relaxation.
We propose to model the decrease in the velocity penetration length $\lambda(\delta)$ using the following expansion:

$$
\lambda=\lambda_{\infty}\left[1+\nu_{1} \frac{\delta^{*}}{\delta_{L}}+\nu_{2}\left(\frac{\delta^{*}}{\delta_{L}}\right)^{2}+\cdots\right],
$$

where $\delta^{*}$ here denotes the onset of slow relaxation (counted, as $\delta_{L}$, from localization). Large- $\delta$ limit of $\lambda$ is $\lambda_{\infty}$, whereas small- $\delta$ limit is expressed as $\lambda_{\infty}\left(1+\nu_{1}+\nu_{2}+\cdots\right)>\lambda_{\infty}$. Within this framework, the coefficient $\nu_{1}$ has to be treated as a decreasing function of $r$ to account for the observed radial evolution of the relaxation process [Fig. 7(b)]. Specifically, for small values of $r$, we should have $\nu_{1}>0$, so that the decrease in $\lambda(\delta)$ is slow, dominated by the first-order $\delta^{*} / \delta_{L}$ term. On the contrary, for large $r$, we expect $\nu_{1}=0$, so that the long-term, stationnary regime is reached quasiinstantaneously at $\delta=\delta^{*}$.

Inserting the above expansion (4) in Eq. (1) yields

$$
\frac{d u_{\theta}}{d \delta}=C_{0} e^{-r / \lambda_{\infty}}+C_{0} \nu_{1}(r) \frac{r}{\lambda_{\infty}} e^{-r / \lambda_{\infty}} \frac{\delta^{*}}{\delta_{L}}+\cdots
$$

For simplicity, we neglected here variations with $\delta$ of the prefactor $C_{0}$. Straightforward integration of Eq. (5) yields an expression for the cumulative displacement $u_{\theta}$ and, taking advantage of the relation $R \gg r \gg \lambda_{\infty}$, the cumulative shear strain $\gamma \approx\left|\partial u_{\theta} / \partial r\right|$. Limiting expansions to the first order in $\delta^{*} / \delta_{L}$, we obtain the following approximation:

$$
\gamma_{L} \approx f_{0}(r)\left(\delta_{L}-\delta^{*}\right)+f_{1}(r) \delta^{*} \ln \left(\frac{\delta_{L}}{\delta^{*}}\right)+\Gamma_{0}(r)
$$

where $f_{0}(r)=C_{0} \exp \left(-r / \lambda_{\infty}\right) / \lambda_{\infty}$,

$$
f_{1}(r)=C_{0}\left(\frac{\nu_{1}}{\lambda_{\infty}}+\left|\frac{d \nu_{1}}{d r}\right|\right) \frac{r}{\lambda_{\infty}} e^{-r / \lambda_{\infty}}
$$

and $\Gamma_{0}(r)$ represents the "initial" value of $\gamma_{L}$ at $\delta=\delta^{*}$.

The first term in Eq. (6) represents the stationnary, longterm increase of $\gamma_{L}$, whereas the second term corresponds to the slow relaxation part. Consistent with Fig. 8, expression (6) indeed predicts, for sufficiently small values of $r$ and $\delta_{L}$, a regime in which the logarithmic increase of $\gamma_{L}\left(\delta_{L}\right)$ is dominant over the linear increase. To check further the validity of this model, we plotted, for low values of $\delta$, the radial profiles of $\left\langle\gamma_{L}\right\rangle_{\theta}$ normalized by $\log \left(\delta_{L} / \delta^{*}\right)$ (Fig. 9). As shown, it is possible to find a unique choice of the parameter $\delta^{*}$ for which all the displayed profiles collapse reasonnably well (indicating that $\Gamma_{0} \approx 0$ ). Furthermore, the radial dependence of $\left\langle\gamma_{L}\right\rangle_{\theta}$ is found in good agreement, over a significant range of $r$, with expression (7) and a constant $\nu_{1}$ (Fig. 9). Actually, though suppressed in Fig. 9, raw profiles generally display a nonzero asymptotic limit for large $\delta$. This is not accounted for by Eq. (6), and can presumably be attributed to corrective terms such as influence of radial displacements, etc. 


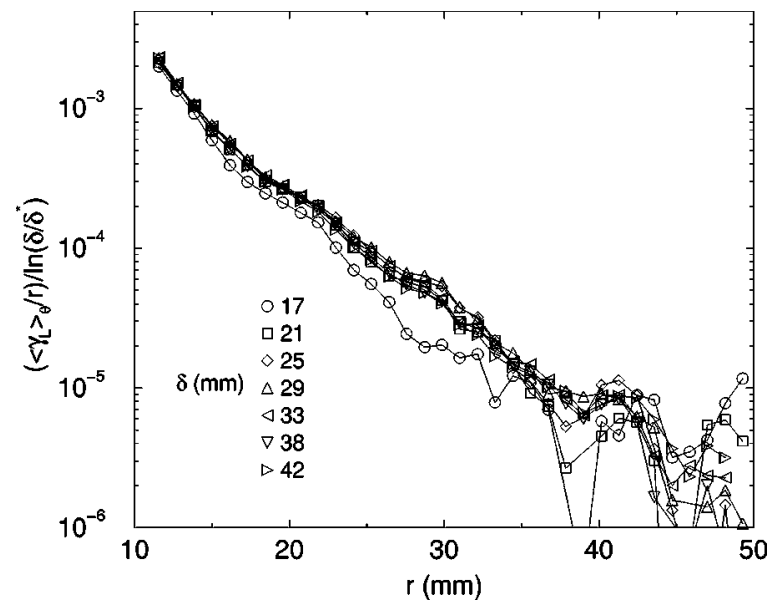

FIG. 9. Radial profiles of the quantity $\left\langle\gamma_{L}\right\rangle_{\theta} / r$ normalized by the slip function $\ln \left(\delta_{L} / \delta^{*}\right)$, for seven (relatively low) values of slip $\delta$ (semilogarithmic scale). Best collapse of the represented profiles is reached for a value of $\delta^{*}=2.3 \mathrm{~mm}$. Good linearity of the curves in the chosen mode of representation indicates that $\gamma_{L}$ $\propto r \exp \left(-r / \lambda_{\infty}\right)$. The order of magnitude of $\lambda_{\infty}$ induced from the slope of the profiles $(\approx 5 \mathrm{~mm})$ is consistent with Fig. 5 . Note that, for all profiles, the asymptotic limit at large $\delta$ has been artificially set to 0 (see text).

\section{DISCUSSION}

\section{A. Four regimes of deformation}

From the previous results, we can identify four different regimes for the deformation of the granular sample. This situation is illustrated by the "phase diagram" in Fig. 10 (see also Fig. 7). Regime I, for $0 \leqslant \delta \leqslant \delta_{1} \approx 10-12 \mathrm{~mm}$, corresponds to the prelocalization deformation of the sample. During this stage, the whole sample undergoes large displacement increments of the same order as $\Delta \delta$. This regime is not stationnary and eventually becomes unstable at localization.

Regime II establishes after localization and before relaxation onset, i.e., for $\delta_{1} \leqslant \delta \leqslant \delta_{2}=\delta_{1}+\delta^{*}$. Displacement and strain increments are greatly reduced inside the bulk (compared to regime I), and velocity follows an exponential radial profile with a penetration length $\lambda \approx 9 \mathrm{~mm}$ (Fig. 5). For large values of $r$, this regime is clearly visible and quasistationnary up to $\delta_{2} \approx 26 \mathrm{~mm}$ [Fig. 7(b)]. On the contrary, for small $r$, regime II is much shorter lived, or even nonexistent, as indicated by the absence of an evident plateau for $\delta \gtrsim 12 \mathrm{~mm}$ in Fig. 6(a), and the small value of $\delta^{*}$ found in Fig. 9 $(\approx 2 \mathrm{~mm})$.

Regime III corresponds to the long-term, stationnary evolution of the bulk. Displacements and strain increments have undergone further, significant reduction since regime II. Velocity profile is still exponential but with a much shorter penetration length: $\lambda_{\infty} \approx 3 \mathrm{~mm}$. As for regime II, regime III can clearly be identified for large values of $r$ in Fig. 7b, but never fully establishes, in the $\delta$ range we investigated, at smaller values of $r$.

Lastly, the fourth regime, confined to small values of $r$, represents the slow transition between regimes II and III. It is characterized by slow, hyperbolic relaxation of the strain in-

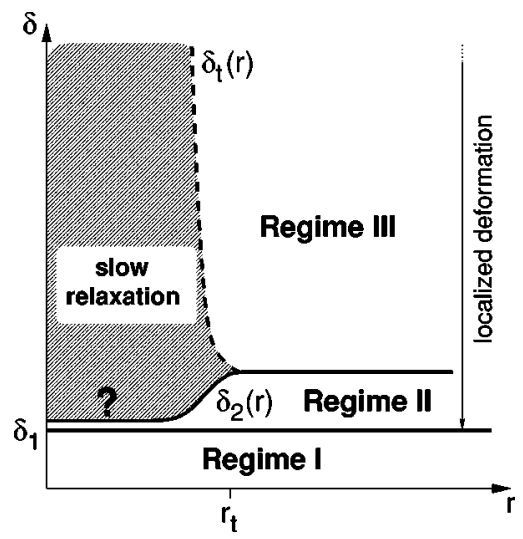

FIG. 10. Diagram picturing the existence domains for the four deformation regimes of the bulk (see text). Full curves represent sharp ("instantaneous") transitions, dashed curves represent soft transitions. These boundaries have been traced qualitatively, their precise shape being unknown. In particular, the question mark concerns the existence of regime II at small values of $r$.

crements (and of the velocity penetration length $\lambda$ ). In Fig. 10 , the boundary $\delta_{t}(r)$ between slow relaxation and regime III actually represents a soft transition. We can also prognosticate that this boundary $\delta_{t}(r)$ should be a relatively steep function of $r$. Indeed, the quality of the spatial fit in Fig. 9 does not appear much dependent on $\delta$. Note that an equation for $\delta_{t}(r)$ can easily be extracted from expression (6):

$$
\delta_{t}=\delta_{2}+\frac{f_{1}(r)}{f_{0}(r)} \delta^{*} \ln \left(\frac{\delta_{t}-\delta_{1}}{\delta_{2}-\delta_{1}}\right) .
$$

As expected, this equation admits a solution $\delta_{t} \neq \delta_{2}$ only for $r$ smaller than a characteristic value $r_{t}$ (Fig. 10). Furthermore, it can also be shown that Eq. (8) predicts a fast growth of the solution $\delta_{t}$ when $r$ decreases.

\section{B. Jamming transition}

The transition between regimes I and II appears well marked in Fig. 7(a). The corresponding reduction in strain increments occurs simultaneously for all $r$ values and, more generally, for all points inside the bulk. This transition thus marks a clear bifurcation in the sample behavior, which is characteristic of a localization process. At localization, the interfacial layer continues to flow while the bulk probably switches to a jammed state (since further displacement increments are far less than a grain size).

In this respect, the analogy between postlocalization deformation in our samples and findings for the static part of avalanching piles is particularly interesting. Following [15], we may suggest that intermittent and heterogeneous velocity clusters, resulting in average in an exponential profile with $r$, do constitute the generic response of a jammed packing sheared by a "flowing" layer. In the same spirit, we note in Fig. 6 that the bulk compacts during regime II and slow relaxation. Various studies have shown that slow compaction is ubiquitous in jammed packings submitted to small excitations $[18,19]$. In our case, the excitation signal triggering 
compaction is probably generated internally by the intermittent bursts of displacement and shear.

\section{Decoupling transition}

As seen, the transition between regimes II and III is marked by a significant decrease with slip of the velocity penetration length $\lambda$. It thus corresponds to a coupling reduction between the interfacial layer and the bulk. A possible reason for such a decoupling could be a sudden change in the bulk sollicitation. Indeed, this could explain the sharpness of the transition for large values of $r$. This also implies that the slow process observed for small $r$ is due to (nonlinear) relaxation mechanisms active locally within this particular part of the bulk. In fact, everything happens as if regions inside the bulk would retain a memory of the strain rates they have undergone previously. Hence, regions at small $r$, where strain rate was higher, need more "time" to adapt to the new coupling mode, and thus relax slower (in average) than regions at large radii where shear was already very low. It is not the first time that memory effects are invoked in combination with slow dynamics for jammed granular systems [20]. Note, however, that the notion of "time" should in our case be replaced by slip: it is ultimately the cylinder rotation that creates fluctuations allowing for small rearrangements inside the bulk and hence for (slow) relaxation.

Interestingly, slow relaxation active at small $r$ has presumably a signature at the macro-scale. Indeed, we described in a previous paper [11] the evolution of the macroscopic torque $\Gamma$ exerted to rotate the inner cylinder. This quantity displays, after localization, a significant and monotonic decrease with slip. Furthermore, this slip-weakening process is very well fitted by a power law in $\delta$, without any characteristic slip scale. The observed macro- and microrelaxations thus present strong similarities. They probably both represent the same effect envisioned at two different scales.

A detailed study of the macroscopic slip weakening points out the role of comminution in our results. In particular, noslip weakening is observed for experimental configurations that prevent comminution inside the interfacial layer (use of a smooth cylinder, or of glass beads). When comminution is present, on the contrary, slip weakening appears during the initial shear of fresh samples as well as after reversals of the cylinder rotation [11]. A possible mechanism is that comminution, acting as a weakening factor for the interfacial layer, triggers a secondary bifurcation inside this zone. This would result in a modification of the velocity profile in the interfacial layer and, consequently, of the "boundary condition" exerted on the bulk. Specifically, we observe for large values of $\delta$ a thin transition layer between the interfacial zone and the bulk (Fig. 2). It is identifiable as a highly compact area where crushed particles fill the porosity between initial particles (layer $t$ ). This well-lubricated layer probably tends to accommodate most of the imposed straining. It then constitutes a decoupling surface between the interfacial zone and the bulk.

\section{CONCLUSIONS}

In conclusion, we have studied the postlocalization strain field outside the shear band in an extended shear experiment.

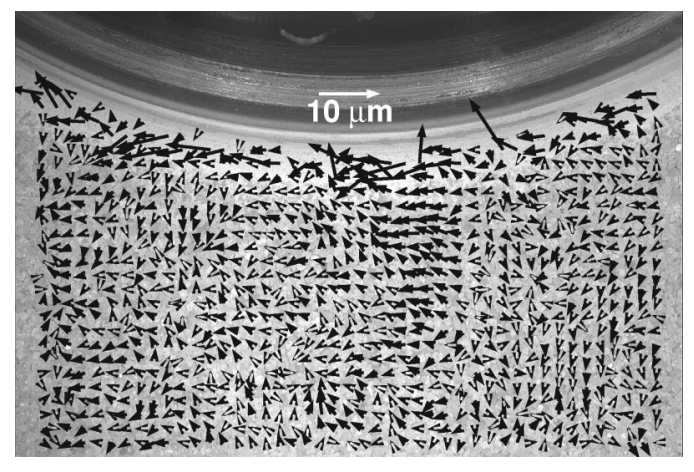

FIG. 11. Differences between computed and imposed displacements (residuals). This result is obtained by testing CIV for recovering a known displacement field-in this case, the field displayed in Fig. 3 (see text).

Though very small compared to strains within the band, this strain field displays the rich dynamics, typical of jammed packings. Large scale patches of coherent strain frequently develop. However, they are fleeting and very intermittent. They are probably the signature of macroclusters of purely mechanical origin, and might denote eddylike structures.

Azimuthally averaged velocity profiles have an exponential shape with radius $r$. Furthermore, the penetration length $\lambda$ of these profiles exhibits a slow decrease with slip $\delta$, denoting a progressive decoupling between the interfacial layer and the bulk. Further investigations using cumulative quantities reveal that slow relaxation is, in fact, essentially concentrated close to the interfacial layer, whereas more distant regions switch quicker to the new coupling mode. We emphasize the role of comminution for this decoupling phenomenon, through the creation of a well-lubricated transition layer between the interfacial and the bulk.

For small radii, relaxation of shear strain is well modeled by a $1 / \delta$ law, without any characteristic length scale. This

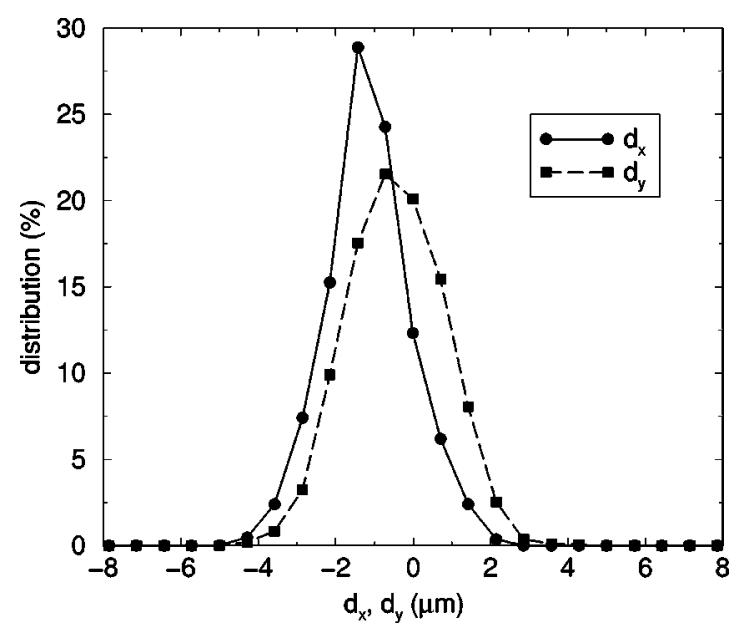

FIG. 12. Statistical distribution of the residuals inside the bulk. Ten tests of synthetic deformation are integrated in this plot, using constant displacement fields. The two curves, respectively, correspond to the cartesian coordinates $x$ and $y$ ("natural" coordinates of CIV) of the residual vector $\mathbf{d}$. Dispersion of the values, at a $95 \%$ confidence level, is $\pm 310^{-2} \mathrm{px}$. 
denotes extremely long rearrangement processes in the packing. Furthermore, this compares qualitatively well with the slow decrease of the macroscopic torque $\Gamma$ in the postlocalization domain. Our results thus provide an important link between the macroscopic friction law of a granular system and microscopic processes active at the grain scale. The detailed understanding of nonlinear slip-weakening effects in friction laws has very important implications, particularly for the physics of earthquakes [21]. Here, we show that, in spite of localization, the portion of the sample effectively involved in the macroscopic response substantially extends outside the shear band. The thickness of this zone, which can be defined from the boundary of the slow relaxation domain in Fig. 10, displays only weak dependence on imposed slip.

\section{APPENDIX: CIV ACCURACY}

CIV is generally applied to a pair of photos representing the same object in two (slightly) different deformation stages. The essence of the technique is to determine the maximum cross correlation between small zones extracted from these two images. This maximum corresponds to the displacement (translation) vector of the considered zone. By moving the zone of interest, it is then possible to determine displacements at various positions inside the photo. A refine- ment of the technique, crucial in our case, allows to achieve subpixel accuracy in this displacement computation. It briefly consists in iterative interpolations of the correlation function [22,23]. For the sake of efficiency, we make an extensive use of the Fourier transforms. Furthermore, to improve the contrast, we evaluate correlations from image gradients rather than from direct gray levels.

In Fig. 11, we show an illustration of CIV precision using a synthetically deformed image. We interpolated the displacement field represented in Fig. 3 (which is itself a CIV output) and used it to deform the digital picture displayed in the background. CIV was then applied between the initial and the deformed images. Note that, due to interpolations, the computed residuals are likely to be overestimated by this procedure. Still, only minor differences show up between the imposed and the computed displacement fields (Fig. 11). In particular, all the characteristic structures of the initial field are very well recovered.

Quantitatively, we calculated CIV accuracy, using the same procedure of synthetic deformation, but with constant displacement fields in order to minimize interpolation effects (Fig. 12). This yields a value of $3 \times 10^{-2}$ px, i.e., $2 \mu \mathrm{m}$. Note that this value slightly depends on the considered series of photos.
[1] M. Ohnaka, M. Akatsu, H. Mochizuki, A. Odedra, F. Tagashira, and Y. Yamamoto, Tectonophysics 277, 1 (1997).

[2] C. Marone, Annu. Rev. Earth Planet Sci. 26, 643 (1998).

[3] B. Cambou, Behaviour of Granular Materials, CISM Courses and Lectures Vol. 385 (Springer-Verlag, New York, 1998).

[4] C.T. Veje, D.W. Howell, and R.P. Behringer, Phys. Rev. E 59, 739 (1999).

[5] D.M. Mueth, G.F. Debregeas, G.S. Karczmar, P.J. Eng, S.R. Nagel, and H.M. Jaeger, Nature (London) 406, 385 (2000).

[6] D.M. Mueth, Phys. Rev. E 67, 011304 (2003).

[7] D. Howell, C.T. Veje, and R.P. Behringer, Phys. Rev. Lett. 82, 5241 (1999).

[8] F. Radjai, M. Jean, J.-J. Moreau, and S. Roux, Phys. Rev. Lett. 77, 274 (1996).

[9] F. Radjai, S. Roux, and J.-J. Moreau, Chaos 9, 544 (1999).

[10] A. Zervos, I. Vardoulakis, M. Jean, and P. Lerat, Mech. Cohesive-Frict. Mater. 5, 305 (2000).

[11] G. Chambon, J. Schmittbuhl, and A. Corfdir, Geophys. Res. Lett. 29, 1366 (2002).

[12] P. Lerat, Thèse de doctorat, École Nationale des Ponts et Chaussées, 1996.

[13] A. Corfdir, P. Lerat, and J.-N. Roux, in Powder and Grains
2001, edited by Y. Kishino, Proceedings of the Fourth International Conference on Micromechanics of Granular Media, Sendai, Japan, 2001 (Balkema, Lisse, The Netherlands, 2001), pp. 315-318.

[14] F. Radjai and S. Roux, Phys. Rev. Lett. 89, 064302 (2002).

[15] T.S. Komatsu, S. Inagaki, N. Nakagawa, and S. Nasuno, Phys. Rev. Lett. 86, 1757 (2001).

[16] B. Andreotti and S. Douady, Phys. Rev. E 63, 031305 (2001).

[17] F. Hild, B. Raka, M. Baudequin, S. Roux, and F. Cantelaube, Appl. Opt. 41, 6815 (2002).

[18] J.B. Knight, C.G. Fandrich, C.N. Lau, H.M. Jaeger, and S.R. Nagel, Phys. Rev. E 51, 3957 (1995).

[19] M. Nicolas, P. Duru, and O. Pouliquen, Eur. Phys. J. E 3, 309 (2000).

[20] C. Josserand, A.V. Tkachenko, D.M. Mueth, and H.M. Jaeger, Phys. Rev. Lett. 85, 3632 (2000).

[21] K. Uenishi and J.R. Rice, J. Geophys. Res. 108, 2042 (2003).

[22] F. Hild, J.-N. Périé, and M. Coret, Internal Report 230, 1999 (unpublished).

[23] L. Chevalier, S. Calloch, F. Hild, and Y. Marco, Eur. J. Mech. A/Solids 20, 169 (2001). 